\title{
Cave Bluttransfusion bei akutem Herzinfarkt
}

\section{Nach der gegenwärtigen Datenlage sind Bluttransfusionen bei Patienten mit akuter koronarer Ischämie und Anämie nicht nur nicht gerechtfer- tigt, sondern sogar schädlich.}

- In einer Metaanalyse von zwölf randomisierten Studien mit 203144 Herzinfarktpatienten wurde der Zusammenhang zwischen Hämoglobinwert und Mortalität untersucht [1]. Als An-ämie galten $\mathrm{Hb}$-Werte unter $13 \mathrm{bzw} .12 \mathrm{~g} / \mathrm{dl}$ bei Männern bzw. Frauen oder die individuelle Definition in den Studien. Mit einer Ausnahme war die Mortalität der anämischen Patienten in allen Studien um das 1,05- bis 8.2-Fache, im Mittel um das 2,08-Fache erhöht.

In einer zweiten Metaanalyse [2] wurden zehn Studien - darunter nur eine randomisierte - mit 203665 Patienten mit akutem Herzinfarkt ausgewer- tet. Bluttransfusionen bei HämoglobinWerten zwischen 8,9 und 13,9 g/dL erhöhten die Mortalität signifikant um das 2,91-Fache und das Risiko für einen ReInfarkt um das 2,04-Fache. Diese Verschlechterung bestand auch bei Patienten mit schwerer Anämie (Hämatokritwerte unter 30\%), war unabhängig von zahlreichen Einflussfaktoren, auch vom Ausgangswert des Hämoglobins, vom niedrigsten $\mathrm{Hb}$-Wert und von der $\mathrm{Hb}$ - Änderung im Verlauf.

\section{Kommentar}

Nach Lawler et al. haben Patienten mit Herzinfarkt und Anämie ein erhöhtes Sterberisiko. Da die Sauerstoffzufuhr für ischämisches Gewebe entscheidend vom $\mathrm{Hb}$-Wert abhängt, erscheint es plausibel, bei anämischen Patienten mit akuter koronarer Ischämie die Sauerstoffzufuhr durch Bluttransfusion zu verbessern. Chatterjee et al. kommen aber aufgrund der Auswertung von - methodisch wenig zuverlässigen - Beobachtungsstudien zu dem Ergebnis, dass Bluttransfusionen bei Patienten mit akutem Herzinfarkt und Anämie die Mortalität nicht etwa senken, sondern erhöhen. Auch das ist plausibel, erhöht doch die Transfusion die Herzbelastung. Da stellt sich die Frage: Was ist gefährlicher für den anämischen Infarktpatienten, die Anämie oder die Bluttransfusion? Nach dem Prinzip „Primum nihil nocere" wird man bei dieser Unsicherheit mit der Indikation für eine Bluttransfusionen sehr zurückhaltend sein, bis Ergebnisse aus zuverlässigen Studien vorliegen.

H. HolzGREVE.

- [1] P. R. Lawler et al.

Anemia and mortality in acute coronary syndromes: A systematic review and meta-analysis. Am. Heart J. 165 (2013) 143-153

- [2] S. Chatterjee et al.

Association of blood transfusion with increased mortality in myocardial infarction. JAMA Intern Med. 173 (2013) 132-139 